\title{
Whole brain radiotherapy using four-field box technique with tilting baseplate for parotid gland sparing
}

\author{
Jaehyeon Park, MD, Ji Woon Yea, MD, PhD \\ Department of Radiation Oncology, Yeungnam University College of Medicine, Daegu, Korea
}

Purpose: The aim of this study is to evaluate the efficacy and feasibility of four-field box whole brain radiotherapy (FB-WBRT) with tilting baseplate by comparing bilateral WBRT (B-WBRT).

Methods and Materials: Between March 2016 and September 2018, 20 patients with brain metastases underwent WBRT using the four-field box technique. WBRT is performed with a dose of $30 \mathrm{~Gy}$ in 10 fractions daily. Two computed tomography simulations per person were performed. One was in the traditional supine position for B-WBRT and the other by applying the tilting acrylic supine baseplate to elevate the head by $40^{\circ}$ for FB-WBRT. The B-WBRT used the field-in-field technique, which is the most commonly used method in our institution. The FB-WBRT comprised anterior, posterior, and bilateral beams. A wedge was applied in anterior and posterior fields to compensate for skull convexity.

Results: The average of Dmean of both parotid glands was 10.2 Gy (range, 3.8 to $17.8 \mathrm{~Gy}$ ) in B-WBRT and 5.4 Gy (range, 2.0 to $11.7 \mathrm{~Gy})$ in FB-WBRT $(p<0.05)$. Compared to B-WBRT, FB-WBRT reduced the mean dose of the right and left parotid glands from $10.1 \mathrm{~Gy}$ to $4.9 \mathrm{~Gy}$ and from $10.4 \mathrm{~Gy}$ to $5.8 \mathrm{G}$, respectively $(p<0.05)$. Further, $V_{51} V_{10}, V_{15}, V_{20}$ and $V_{25}$ for the parotid gland decreased significantly in FB-WBRT $(p<0.05)$. The Dmax and Dmean of lens decreased according to the dose-volume histogram.

Conclusion: Compared to B-WBRT, FB-WBRT with a tilting baseplate is a simple and effective method that takes feature of noncoplanar beam to protect the parotid gland.

Keywords: Whole brain radiotherapy, Four-field box technique, Brain metastases, Parotid gland sparing

\section{Introduction}

In South Korea, the incidence of patients with brain metastasis from all malignancies is 5.0 per 1,000 person-years, and the order of major primary sites based on decreasing frequency is lung, liver, breast, and colon [1]. Whole brain radiotherapy (WBRT) is widely used for palliative aim in patients with multiple brain metastases and for prophylactic aim in patients with small cell lung cancer in complete remission after the primary treatment [2-7]. Because the outcomes of patients treated with WBRT is generally poor, the toxicity has been neglected. However, survival is longer owing to the development of cancer treatment and the quality of life is become important [8].

The use of parallel opposed fields for WBRT is a simple and effective method to cover the entire brain. Computed

Received 17 January 2019, Revised 22 February 2019, Accepted 12 March 2019.

Correspondence: Ji Woon Yea, MD, PhD, Department of Radiation Oncology, Yeungnam University College of Medicine, 170 Hyeonchung-ro, Nam-gu, Daegu 42415, Korea. Tel: +82-53-620-3371, Fax: +82-53-624-3599, E-mail: yjw1160@ynu.ac.kr (http://orcid.org/0000-0002-7699-342X)

(c) This is an Open Access article distributed under the terms of the Creative Commons Attribution Non-Commercial License (http://creativecommons.org/ licenses/by-nc/4.0/) which permits unrestricted non-commercial use, distribution, and reproduction in any medium, provided the original work is properly cited.

www.e-roj.org 

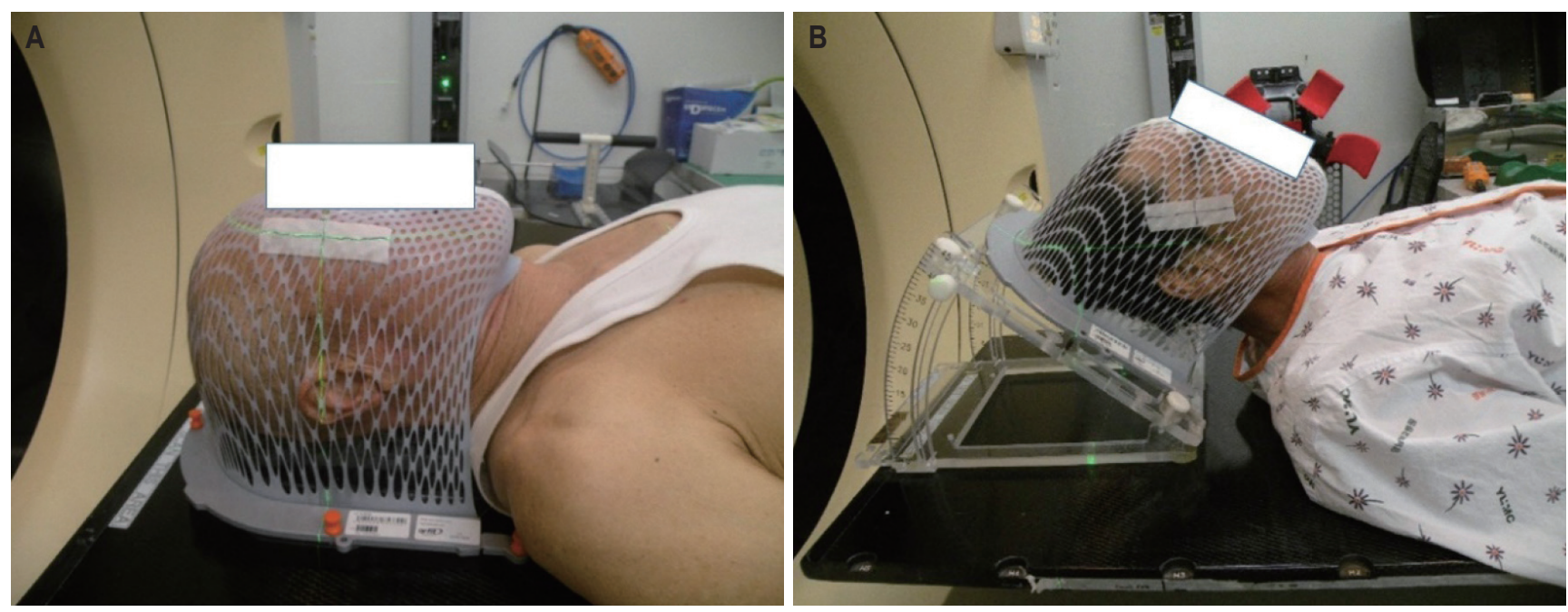

Fig. 1. Patient's position of computed tomography simulation for (A) bilateral whole brain radiotherapy and (B) four-field box whole brain radiotherapy.

tomography (CT) simulation enables the dose distribution of normal organs to be evaluated, although it did not change significantly from two-dimensional radiotherapy (2D-RT) to three-dimensional conformal radiotherapy (3D-CRT). It also made for us to find the large amount volume of parotid glands irradiated during WBRT. Noh et al. [9] recently demonstrated through a normal tissue complication probability model that the parotid gland should be considered as an organ at risk (OAR) during WBRT.

Several researchers have attempted to reduce the dose of the parotid gland, primarily by modifying the lower margin of the treatment field $[10,11]$. Modifying the field has shown good results in protecting the parotid glands, but the target coverage is unclear. We have attempted to reduce the irradiated dose of the parotid gland by adding a superior anterior beam and demonstrated that it could block the parotid gland effectively without jeopardizing the target coverage [12]. However, using a non-coplanar beam could render the treatment process cumbersome. Inspired by this technique, we applied a four-field box technique for WBRT after a patient generated a posture with his head forward. The aim of this study is to evaluate the efficacy and feasibility of four-field box WBRT (FB-WBRT) by comparing it with bilateral WBRT (B-WBRT).

\section{Materials and Methods}

Between March 2016 and September 2018, 20 patients with brain metastases underwent WBRT using the four-field box technique. The informed consent was waived. The median age of the patients was 61 years (range, 47 to 87 years). The primary tumor was from lung cancer (non-small cell lung cancer in 12 patients and small cell lung cancer in 7) and one patient had breast cancer. Two CT simulations per person were performed. One was in the traditional supine position for B-WBRT and the other was applied with a tilting acrylic supine baseplate (MedTec-Civco, Orange City, IW, USA) to elevate his head by $40^{\circ}$ for FB-WBRT, using a thermoplastic mask for immobilization (Fig. 1). We obtained the CT scan images of $5-\mathrm{mm}$ slice thickness, and contoured the OARs, including both the parotid glands and lenses. The brain contours were identified by auto-segmentation of the Eclipse Treatment Planning System 8.6 (Varian Medical System, Palo Alto, CA, USA). The clinical target volume (CTV) included the brain parenchyma and the spinal cord up to the lower level of the atlas, as has expanded by $5 \mathrm{~mm}$ in all directions to create the planning target volume (PTV).

B-WBRT plan was subsequently generated using the fieldin-field technique for reducing the maximum dose, and to increase dose homogeneity [12]. The beam direction for FBWBRT consisted of anterior, posterior, and bilateral beams (Fig. 2). Enhanced dynamic wedge was applied in the anterior and posterior fields to compensate for skull convexity. A suitable wedge angle of $15^{\circ}$ to $30^{\circ}$ was selected for each patient. According to our institutional practice, WBRT was administered using the schedule of $30 \mathrm{~Gy}$ in 10 fractions, for 5 days a week. Electronic portal imaging was performed to obtain images on the anterior and right at the first and 6th days of treatment. 

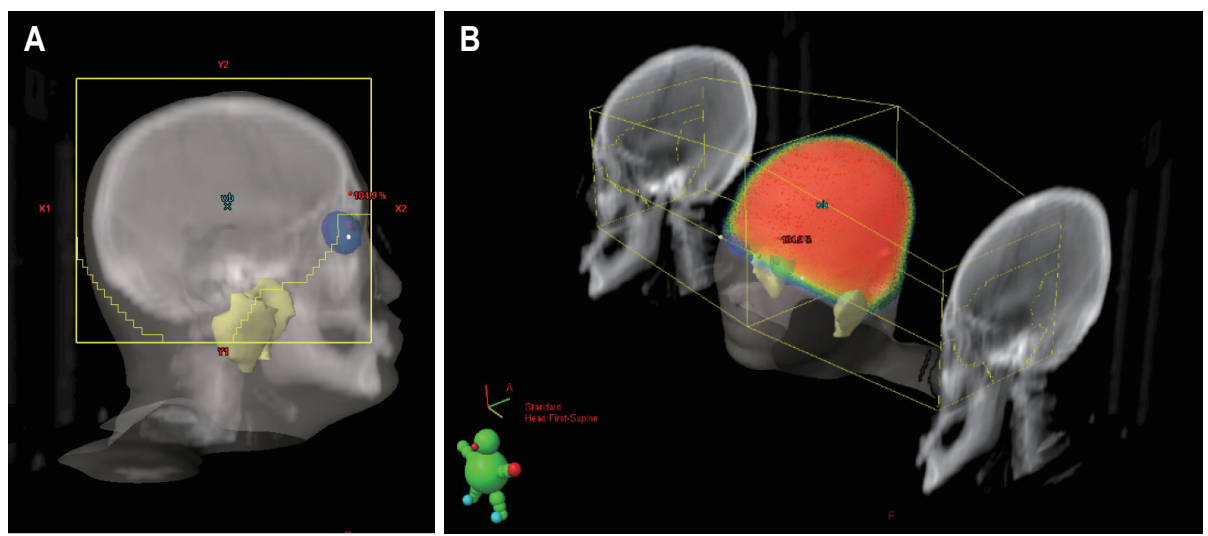

Fig. 2. Beam's eye view (A) right field and (B) fields set up in bilateral whole brain radiotherapy; (C) anterior, (D) right field, and (E) fields set up in four-field box whole brain radiotherapy.
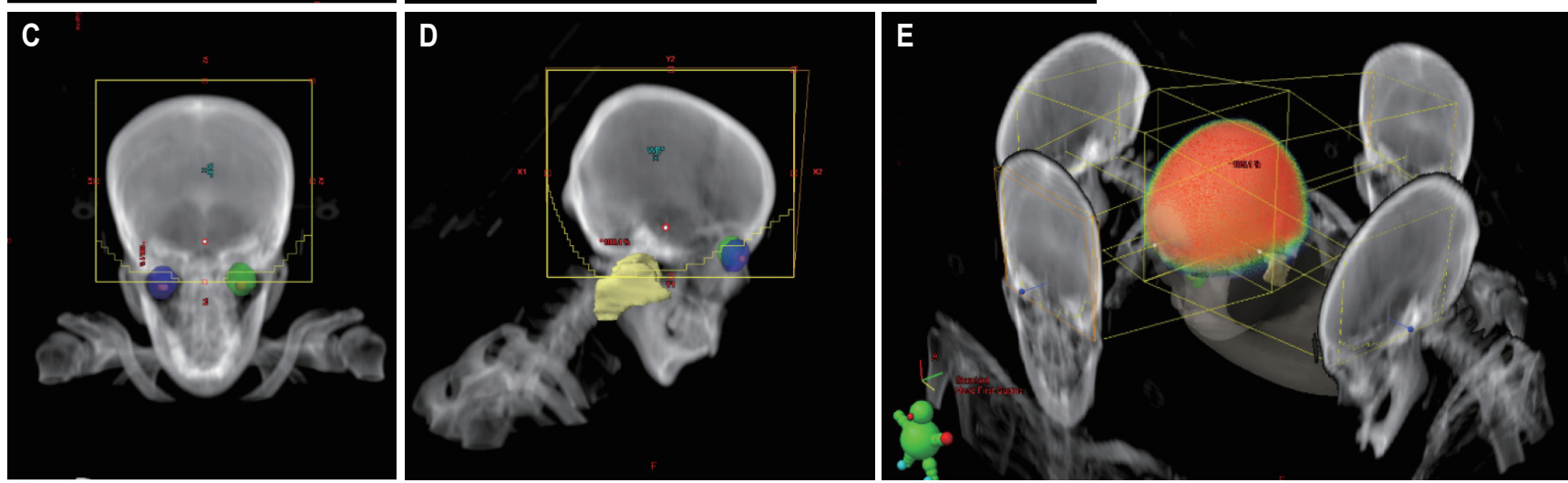

The conformity index $(\mathrm{Cl})$ and homogeneity index ( $\mathrm{HI})$ were utilized in the treatment plan analysis. The $\mathrm{Cl}$ is defined as the ratio of the PTV that receives 95\% of the prescribed dose to the entire PTV, while the $\mathrm{HI}$ is the ratio of the maximum target dose to the prescribed dose. Wilcoxon-signed rank tests were used to compare the dosimetric outcomes, including the dose coverage and OAR doses between the B- and FB-WBRT plans. All the statistical analyses were performed using the SPSS version 21.0 (SPSS Inc., Chicago, IL, USA) software package.

\section{Results}

The mean volume of the right parotid, left parotid, and both parotids were $29.7 \pm 13.7 \mathrm{~cm}^{3}, 28.1 \pm 11.2 \mathrm{~cm}^{3}$, and $57.8 \pm$ $25.1 \mathrm{~cm}^{3}$ in B-WBRT, respectively; $29.5 \pm 14.6 \mathrm{~cm}^{3}, 28.4 \pm 12.5$ $\mathrm{cm}^{3}$, and $58.0 \pm 26.6 \mathrm{~cm}^{3}$ in FB-WBRT, respectively. The dosevolume statistics of the parotid glands are summarized in Table 1, and Fig. 3 demonstrates the dose-volume histogram (DVH). The Dmean of both parotid glands is $10.2 \mathrm{~Gy}$ (range, 3.8 to $17.8 \mathrm{~Gy}$ ) in B-WBRT and 5.4 Gy (range, 2.0 to $11.7 \mathrm{~Gy}$ ) in FBWBRT ( $p$ 0.05). Compared to B-WBRT, FB-WBRT reduced the mean dose of the right and left parotid glands from $10.1 \mathrm{~Gy}$ to 4.9 Gy and from 10.4 Gy to $5.8 \mathrm{~Gy}$, respectively $(p<0.05)$.
Further, $V_{5}, V_{10}, V_{15}, V_{20}$, and $V_{25}$ for the parotid gland decreased significantly in FB-WBRT ( $p<0.05$ ) (Fig. 4).

The Dmax of the lens was $7.3 \pm 3.4$ Gy on the right and 7.6 $\pm 4.6 \mathrm{~Gy}$ on the left in B-WBRT, and $5.0 \pm 2.3$ Gy on the right and $5.0 \pm 2.3 \mathrm{~Gy}$ on the left in FB-WBRT $(p<0.05)$. The Dmean of the lens was $4.2 \pm 2.1 \mathrm{~Gy}$ vs. $3.0 \pm 1.1 \mathrm{~Gy}$ on the right, and 4.2 \pm 2.1 Gy vs. $3.0 \pm 1.1$ Gy on the left in the B-WBRT and NCWBRT plans, respectively $(p<0.05)$.

The dose distribution of both plans is shown in Fig. 5 . The $\mathrm{Cl}$ in B-WBRT and FB-WBRT were $0.86 \pm 0.06$ and $0.85 \pm 0.05$ respectively. The $\mathrm{HI}$ was $1.05 \pm 0.01$ in both plans. There was no statistical significance between both plans.

\section{Discussion and Conclusion}

Parotid sparing has been a major concern in radiation therapy for head and neck cancer because xerostomia is the most typical and uncomfortable side effect. The impairment of parotid gland function could cause problems such as poor dental hygiene, oral infections, and difficulties in chewing and swallowing $[13,14]$. To avoid severe xerostomia, the Quantitative Analyses of Normal Tissue Effects in the Clinic guidelines recommend that the mean dose to at least one 
Table 1. Dose-volume statistics of parotid gland between B-WBRT and FB-WBRT

\begin{tabular}{|c|c|c|c|}
\hline Variable & B-WBRT & FB-WBRT & $p$-value \\
\hline \multicolumn{4}{|l|}{ Parotid right } \\
\hline$V_{5}(\%)$ & $49.0 \pm 16.1$ & $28.8 \pm 15.9$ & $0.001^{*}$ \\
\hline$V_{10}(\%)$ & $38.6 \pm 14.6$ & $17.5 \pm 13.1$ & $0.001^{*}$ \\
\hline$V_{15}(\%)$ & $31.1 \pm 13.2$ & $8.8 \pm 10.4$ & $0.000^{*}$ \\
\hline$V_{20}(\%)$ & $24.0 \pm 11.3$ & $3.9 \pm 7.7$ & $0.000^{*}$ \\
\hline$V_{25}(\%)$ & $15.2 \pm 8.3$ & $1.9 \pm 4.7$ & $0.000^{*}$ \\
\hline $\mathrm{D}_{\min }(\mathrm{cGy})$ & $73.1 \pm 24.3$ & $62.5 \pm 24.6$ & $0.010^{*}$ \\
\hline $\mathrm{D}_{\max }(\mathrm{cGy})$ & $2,939.9 \pm 70.1$ & $2,339.8 \pm 552.5$ & $0.000^{*}$ \\
\hline $\mathrm{D}_{\text {mean }}(\mathrm{cGy})$ & $1,011.4 \pm 339.9$ & $485.8 \pm 285.8$ & $0.000^{*}$ \\
\hline Volume (mL) & $29.7 \pm 13.7$ & $29.5 \pm 14.6$ & 0.151 \\
\hline \multicolumn{4}{|l|}{ Parotid left } \\
\hline$V_{5}(\%)$ & $50.2 \pm 18.0$ & $34.0 \pm 16.7$ & $0.005^{*}$ \\
\hline$V_{10}(\%)$ & $39.5 \pm 17.2$ & $22.1 \pm 14.1$ & $0.002^{*}$ \\
\hline$V_{15}(\%)$ & $32.1 \pm 16.1$ & $11.9 \pm 11.2$ & $0.001^{*}$ \\
\hline$V_{20}(\%)$ & $25.1 \pm 14.3$ & $5.7 \pm 8.5$ & $0.000^{*}$ \\
\hline$V_{25}(\%)$ & $16.4 \pm 10.9$ & $2.6 \pm 5.1$ & $0.000^{*}$ \\
\hline$D_{\min }(c G y)$ & $73.8 \pm 28.1$ & $65.7 \pm 19.9$ & 0.247 \\
\hline $\mathrm{D}_{\max }(\mathrm{cGy})$ & $2,927.3 \pm 98.7$ & $2,463.3 \pm 541.1$ & $0.002^{*}$ \\
\hline $\mathrm{D}_{\text {mean }}(\mathrm{cGy})$ & $1,038.6 \pm 409.3$ & $582.5 \pm 288.6$ & $0.001^{*}$ \\
\hline Volume (mL) & $28.1 \pm 11.8$ & $28.4 \pm 12.5$ & 0.232 \\
\hline \multicolumn{4}{|l|}{ Parotid both } \\
\hline$V_{5}(\%)$ & $49.6 \pm 16.3$ & $32.2 \pm 15.6$ & $0.002^{*}$ \\
\hline$V_{10}(\%)$ & $39.1 \pm 15.1$ & $20.4 \pm 13.1$ & $0.001^{*}$ \\
\hline$V_{15}(\%)$ & $31.6 \pm 13.9$ & $10.8 \pm 10.6$ & $0.000^{*}$ \\
\hline$V_{20}(\%)$ & $24.6 \pm 12.2$ & $4.9 \pm 7.5$ & $0.000^{*}$ \\
\hline$V_{25}(\%)$ & $15.7 \pm 9.1$ & $2.3 \pm 4.8$ & $0.000^{*}$ \\
\hline$D_{\min }(c G y)$ & $66.2 \pm 13.6$ & $60.9 \pm 23.9$ & 0.135 \\
\hline$D_{\max }(c G y)$ & $2,957.2 \pm 57.0$ & $2,550.3 \pm 465.6$ & $0.001^{*}$ \\
\hline $\mathrm{D}_{\text {mean }}(\mathrm{cGy})$ & $1,023.7 \pm 357.3$ & $543.9 \pm 278.7$ & $0.000^{*}$ \\
\hline Volume (mL) & $57.8 \pm 25.1$ & $58.0 \pm 26.6$ & 0.225 \\
\hline
\end{tabular}

Values are presented as mean \pm standard deviation.

B-WBRT, bilateral whole brain radiation therapy; FB-WBRT, four-field box whole brain radiation therapy.

${ }^{*} p<0.05$, statistically significance.

parotid gland should be less than $20 \mathrm{~Gy}$, or that the mean dose to the combined volume of both glands should be less than 25 Gy [15].

The use of parallel opposed fields is the most used method because it provides good target coverage. It includes the brain tissue, skull, and spinal cord to the lower level of the atlas. However, Trignani et al. [16] compared 2D-RT with 3D-CRT for WBRT and discovered that $28 \%$ of patients received a mean dose of $>20 \mathrm{~Gy}$ in the parotid gland. Meanwhile, Noh et al. [9] reported that mean parotid doses of $\geq 20$ and $\geq 25$
Gy were observed in $34.4 \%$ and $6.3 \%$ of 64 individual glands, respectively. Burlage et al. [17] reported significantly reduced salivary flow rates during the first 2 weeks of RT. The flow rate has decreased by approximately $80 \%$ of the initial flow; subsequently, the total cumulative dose was 20 Gy. Given the typical treatment schemes of WBRT that is 30 Gy in 10 fractions, it appears necessary to make attempt to reduce the parotid dose for WBRT.

Efforts have been made to reduce the irradiation dose of the parotid gland. Fiorentino et al. [11] reported excellent 


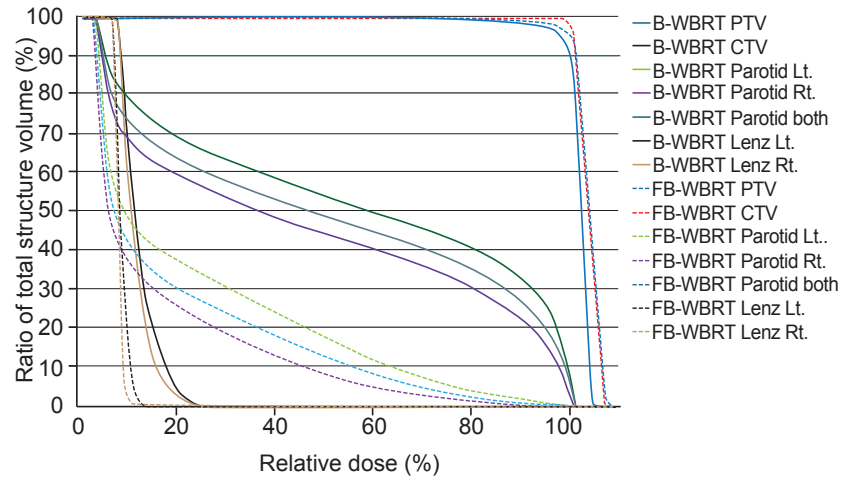

Fig. 3. Dose-volume histogram of parotid gland for bilateral whole brain radiotherapy (B-WBRT) and four-field box whole brain radiotherapy (FB-WBRT). PTV, planning target volume; CTV, clinical target volume.

outcomes in which the medians $\mathrm{V}_{20}$ and $\mathrm{V}_{25}$ of the right parotid gland were 3.5\% (range, $0 \%$ to $44.5 \%$ ) and 1.85\% (range, $0.32 \%$ to $18 \%)$, respectively; further, for the left parotid gland, the medians $V_{20}$ and $V_{25}$ were $3.1 \%$ (range, $0 \%$ to $44.5 \%$ ) and $1.8 \%$ (range, 0\% to $32.2 \%$ ), respectively. They have reduced the treatment field to encompass only the brain tissue and skull. Cho et al. [10] demonstrated the effectiveness in protecting the parotid gland using a modified field technique that is customized to a lower margin around the parotid gland region. The mean dose of the parotid gland was $17.4 \mathrm{~Gy}$ vs. $8.7 \mathrm{~Gy}$, and the $V_{20}$ was $48.4 \%$ vs. $18.2 \%$ in the conventional and modified fields, respectively.

Our previous study using non-coplanar beams demonstrated satisfactory results in which the Dmean of the parotid gland was 13.7 Gy and the $V_{20}$ was $36.4 \%$ in non-coplanar WBRT without compromising the treatment field [12]. Although WBRT with non-coplanar is effective in sparing the parotid gland, we have devised a simpler method that yields the characteristics of the non-coplanar beam. By elevating the patient's head, the anterior beam exhibits a non-coplanarlike effect and the posterior beam is available for use. We anticipated that the dosimetric outcomes in FB-WBRT to be similar to that of WBRT using non-coplanar beams. However, the average of Dmean and the $V_{20}$ of the parotid gland were 5.4 Gy and $4.9 \%$ in FB-WBRT, respectively. These results are better than our expectations. These differences can be attributed to the difference in the PTV margin. This study used a 5-mm margin to create the PTV while our previous study used a 7-mm margin to create the PTV. These changes appear to exhibit a similar effect to the modified field of Cho's study.
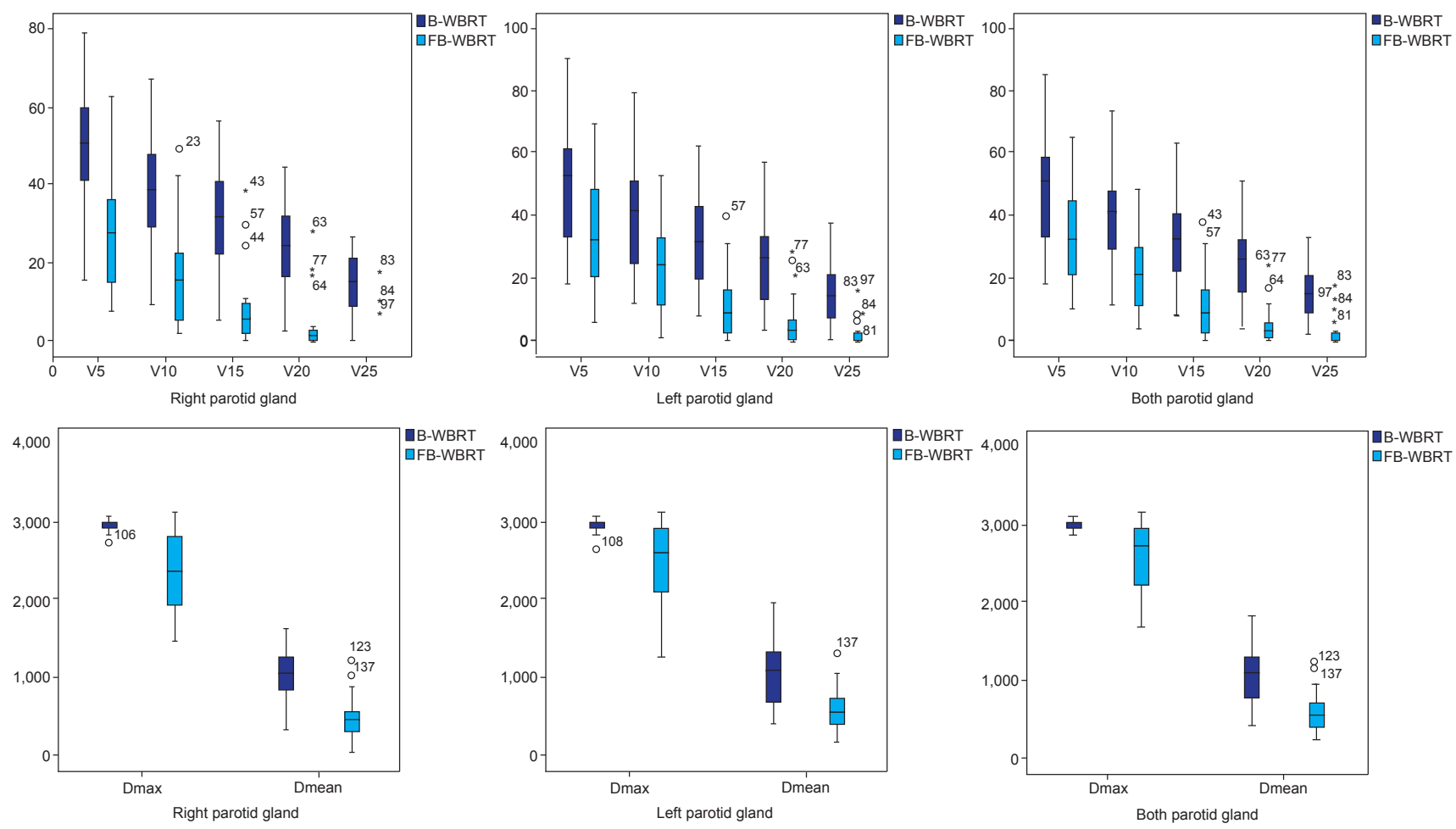

Fig. 4. Boxplots of Dmax, Dmean, and $V_{5}-V_{25}$ for parotid gland. B-WBRT, bilateral whole brain radiation therapy; FB-WBRT, four-field box whole brain radiation therapy. 

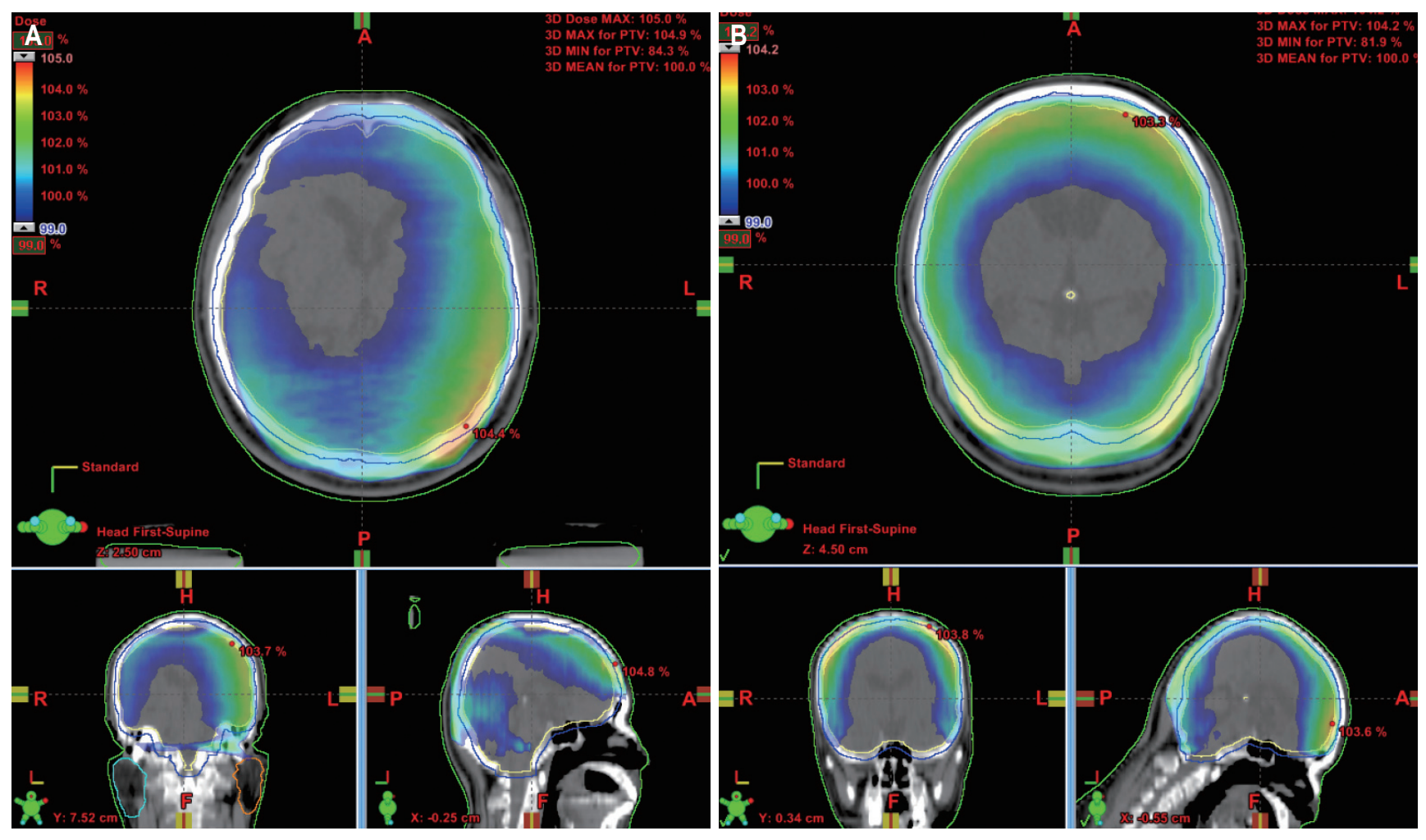

Fig. 5. An example of dose distribution on (A) bilateral whole brain radiotherapy and (B) four-field box whole brain radiotherapy.

Through the dosimetric outcomes of B-WBRT, this assumption is reasonable.

As shown in Fig. 5, FB-WBRT demonstrated a relatively uniform dose distribution in the brain region. However, there was no significant difference in the $\mathrm{Cl}$ and $\mathrm{HI}$. These results were caused by the out-region of the PTV in the posterior neck region being irradiated in FB-WBRT and the field-in-field technique that reduced the hot region in B-WBRT. The Dmax at point of B-WBRT was showed comparable with that of FBWBRT in our study $(104.3 \% \pm 0.95 \%$ vs. $104.9 \% \pm 0.70 \% ; p=$ 0.06).

Intensity-modulated radiotherapy (IMRT) has been widely used in head and neck cancer as it has been proven effective by several prospective trials and systemic reviews for parotid preservation [18-22]. The use of IMRT in WBRT is primarily focused on hippocampal sparing for preserving a patient's neurocognitive function [23-25]. To the best of our knowledge, studies on parotid gland sparing in WBRT using IMRT are scarce. Considering the characteristics of IMRT, the use of IMRT for WBRT could decrease the irradiated dose to the parotid gland. IMRT is generally required a higher workload than 3D-CRT. Recently, the development of RT technique has shortened the time required for treatment planning, quality-assurance and beam delivery and could be overcome the drawback of IMRT [26-28]. However, the advanced RT technique has increased cost $[29,30]$. Therefore, 3D-CRT is advantageous in terms of cost-effectiveness and it is meaningful attempt to reduce the dose for the parotid gland in 3D-CRT for improving the patient's quality of life.

In conclusion, a recent study has shown that xerostomia occurred significantly at the end of WBRT and appeared to be persistent [31]. Therefore, an effort to minimize parotid gland damage in WBRT is necessary. Compared to B-WBRT, FB-WBRT with a tilting acrylic supine baseplate was a simpler and more effective method to take feature of non-coplanar beam for protecting the parotid gland.

\section{Conflict of Interest}

No potential conflict of interest relevant to this article was reported.

\section{References}

1. Kim T, Song C, Han JH, et al. Epidemiology of intracranial metastases in Korea: a national cohort investigation. Cancer 
Res Treat 2018;50:164-74.

2. Coia $L R$. The role of radiation therapy in the treatment of brain metastases. Int J Radiat Oncol Biol Phys 1992;23:229-38.

3. Agboola 0 , Benoit $B$, Cross $P$, et al. Prognostic factors derived from recursive partition analysis (RPA) of Radiation Therapy Oncology Group (RTOG) brain metastases trials applied to surgically resected and irradiated brain metastatic cases. Int J Radiat Oncol Biol Phys 1998;42:155-9.

4. Auperin A, Arriagada R, Pignon JP, et al. Prophylactic cranial irradiation for patients with small-cell lung cancer in complete remission: Prophylactic Cranial Irradiation Overview Collaborative Group. N Engl J Med 1999;341:476-84.

5. Gaspar LE, Scott C, Murray K, Curran W. Validation of the RTOG recursive partitioning analysis (RPA) classification for brain metastases. Int J Radiat Oncol Biol Phys 2000;47:10016.

6. Shaw EG, Su JQ, Eagan RT, Jett JR, Maksymiuk AW, Deigert FA. Prophylactic cranial irradiation in complete responders with small-cell lung cancer: analysis of the Mayo Clinic and North Central Cancer Treatment Group data bases. J Clin Oncol 1994;12:2327-32.

7. Zhang $W$, Jiang $W$, Luan L, Wang $L$, Zheng $X$, Wang G. Prophylactic cranial irradiation for patients with small-cell lung cancer: a systematic review of the literature with metaanalysis. BMC Cancer 2014;14:793.

8. Rohan EA, Miller N, Bonner F 3rd, et al. Comprehensive cancer control: promoting survivor health and wellness. Cancer Causes Control 2018;29:1277-85.

9. Noh OK, Chun M, Nam SS, et al. Parotid gland as a risk organ in whole brain radiotherapy. Radiother Oncol 2011;98:223-6.

10. Cho O, Chun M, Park SH, et al. Parotid gland sparing effect by computed tomography-based modified lower field margin in whole brain radiotherapy. Radiat Oncol J 2013;31:12-7.

11. Fiorentino A, Chiumento $C$, Caivano $R$, et al. "Whole brain radiotherapy: are parotid glands organs at risk?" Radiother Oncol 2012;103:130-1.

12. Park J, Park JW, Yea JW. Non-coplanar whole brain radiotherapy is an effective modality for parotid sparing. Yeungnam Univ J Med 2019;36:36-42.

13. Vissink A, Jansma J, Spijkervet FK, Burlage FR, Coppes RP. Oral sequelae of head and neck radiotherapy. Crit Rev Oral Biol Med 2003;14:199-212.

14. Lal $P$, Bajpai $R$, Khurana $R$, Das KJ, et al. Changes in salivary flow rates in head and neck cancer after chemoradiotherapy. J Cancer Res Ther 2010;6:458-62.

15. Deasy JO, Moiseenko V, Marks L, Chao KS, Nam J, Eisbruch A. Radiotherapy dose-volume effects on salivary gland function.
Int J Radiat Oncol Biol Phys 2010;76(3 Suppl):S58-63.

16. Trignani $M$, Genovesi $D$, Vinciguerra $A$, et al. Parotid glands in whole-brain radiotherapy: $2 D$ versus $3 D$ technique for no sparing or sparing. Radiol Med 2015;120:324-8.

17. Burlage FR, Coppes RP, Meertens $H$, Stokman MA, Vissink A Parotid and submandibular/sublingual salivary flow during high dose radiotherapy. Radiother Oncol 2001;61:271-4.

18. Ghosh-Laskar S, Yathiraj PH, Dutta D, et al. Prospective randomized controlled trial to compare 3-dimensional conformal radiotherapy to intensity-modulated radiotherapy in head and neck squamous cell carcinoma: long-term results. Head Neck 2016;38 Suppl 1:E1481-7.

19. Peng G, Wang T, Yang KY, et al. A prospective, randomized study comparing outcomes and toxicities of intensitymodulated radiotherapy vs. conventional two-dimensional radiotherapy for the treatment of nasopharyngeal carcinoma. Radiother Oncol 2012;104:286-93.

20. Gupta T, Agarwal J, Jain S, et al. Three-dimensional conformal radiotherapy (3D-CRT) versus intensity modulated radiation therapy (IMRT) in squamous cell carcinoma of the head and neck: a randomized controlled trial. Radiother Oncol 2012;104:343-8.

21 Nutting CM, Morden JP, Harrington KJ, et al. Parotid-sparing intensity modulated versus conventional radiotherapy in head and neck cancer (PARSPORT): a phase 3 multicentre randomised controlled trial. Lancet Oncol 2011;12:127-36.

22. Gupta T, Kannan S, Ghosh-Laskar S, Agarwal JP. Systematic review and meta-analyses of intensity-modulated radiation therapy versus conventional two-dimensional and/or or threedimensional radiotherapy in curative-intent management of head and neck squamous cell carcinoma. PLoS One 2018;13:e0200137.

23. Chung $E$, Noh JM, Lee $K C$, et al. Dummy run of quality assurance program before prospective study of hippocampussparing whole-brain radiotherapy (HS-WBRT) and simultaneous integrated boost (SIB) for multiple brain metastases from non-small cell lung cancer: Korean Radiation Oncology Group (KROG) 17-06 study. Cancer Res Treat 2018 Oct 15 [Epub]. http://doi.org/10.4143/crt.2018.415.

24. Gondi V, Tolakanahalli R, Mehta MP, et al. Hippocampalsparing whole-brain radiotherapy: a "how-to" technique using helical tomotherapy and linear accelerator-based intensity-modulated radiotherapy. Int J Radiat Oncol Biol Phys 2010;78:1244-52.

25. Oehlke O, Wucherpfennig D, Fels F, et al. Whole brain irradiation with hippocampal sparing and dose escalation on multiple brain metastases: Local tumour control and survival. 
Strahlenther Onkol 2015;191:461-9.

26 Hall WA, Fox TH, Jiang $X$, et al. Treatment efficiency of volumetric modulated arc therapy in comparison with intensity-modulated radiotherapy in the treatment of prostate cancer. J Am Coll Radiol 2013;10:128-34.

27. Mashhour K, Kamaleldin M, Hashem W. RapidArc vs conventional IMRT for head and neck cancer irradiation: is faster necessary better? Asian Pac J Cancer Prev 2018;19:20711.

28. Yan H, Dai JR, Li YX. A fast optimization approach for treatment planning of volumetric modulated arc therapy.
Radiat Oncol 2018;13:101.

29. Paravati AJ, Boero IJ, Triplett DP, et al. Variation in the cost of radiation therapy among Medicare patients with cancer. J Oncol Pract 2015;11:403-9.

30. Van de Werf E, Verstraete J, Lievens Y. The cost of radiotherapy in a decade of technology evolution. Radiother Oncol 2012;102:148-53.

31. Wang K, Pearlstein KA, Moon DH, et al. Assessment of risk of xerostomia after whole-brain radiation therapy and association with parotid dose. JAMA Oncol 2018 Nov 29 [Epub]. http://doi.org/10.1001/jamaoncol.2018.4951. 